\title{
NOTES ON THE ANALYSIS OF BUTTER.
}

Br J. M. Milne, Ph.D.

Read before the Society of Public Analysts, on 15th_January, 1879.

Harrsg lately had occasion to examine some samples of butter, and having made a number of experiments in connection therewith, it may not be out of place to bring the results obtained under the notice of the Society.

Though these notes contain nothing essentially new, still as the present process for butter analysis is of comparatively recent date, $I$ think it is well that as many as possible of the results obtained by different analysts should be published; and, furthermore, there are one or two little modifications which I have found useful, and which may possibly be of adrantage to others.

The process employed was the modification of Muter's original method, described by Jones, in The ANaryst.* The standard solutions we made as there directed; but latterly, instead of decinormal soda, as recommended by him, the semi-normal soda used for nitrogen determinations was employed, and found to give quite as accurate results, and, of course, obviating the necessity of multiplying standard solutions.

I can bear testimony to the ease with which the butter-fat can be saponified com- 
pletely, on the top of an ordinary water oven, when the flask and its contents are pretty frequently shaken, and provided not much more than five grams of the fat are used for each experiment. As a means of preparing the fat for analysis, the method of simply melting the butter, allowing to subside for a short time, and then filtering, seems to leave nothing to be desired. That the fat gains in weight when kept melted for a length of time at $100^{\circ} \mathrm{C}$, or when heated above that temperature, is, I think, indisputable. A weighed quantity of fat was placed in a small basin, in the water oven, with the following result :-

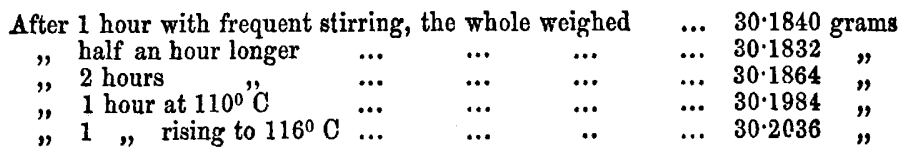

In determining the water in the original butter, a constant weight is possible to be obtained if a moderate quantity of the sample is placed in a very flat platinum basin, on an open water bath, and weighed at short intervals. When the basin and contents were kept in a olose oven, it was found very difficult to obtain a constant weight. It has since occurred to me that it would be of great advantage to dry the fatty acids obtained in the analysis, on the open water bath also.

The trouble and difficulty experienced in weighing out approximately five grams of the butter fat, more or less, for saponification, may be reduced to a minimum, by the following simple expedient. The melted fat is run into a small stoppered Schuster's burette, and the tubulation having been carefully cleaned from adhering fat, the stopper is replaced, and the weight of the whole ascertained and noted. An experiment is first made in order to find approximately the number of drops of fat, which must be transferred from the particular burette used by the operator, into a tared vessel, in order to make up five grams, and this number is also noted. With the burette used in these experiments it was found that $280 *$ drops of butter-fat were required for five grains by weight. In practice it is only necessary to weigh the burette and contents (while fluid), loosen the stopper, and drop the required number of drops into the flask in which the fat is to be saponified. A drop or two may then be added in excess of this number in order to have the amount taken slightly over five grams, and the burette and contents are again weighed, to ascertain the exact weight of fat used. There are several advantages attached to this method of procedure. The fat remains longer fluid than in an open beaker. The wished for amount can be quickly obtained, and with ordinary care the neck of the saponifying flask is not soiled, and the fat has no tendency to run down the outside of the burette-ar annoyance only too common when beakers, \&c., are used-and even when a drop of fat does get outside the narrow orifice of the burette it very soon solidifies and remains stationary.

As regards the treatment and drying of the fatty acids, I have found the following very convenient:-After washing in the manner recommended by Jones, the cake of fatty acids is broken into small pieces. This is easily accomplished by a few taps on the flask, and there is then no difficulty in transferring the pieces to the filter. One or two judicious rinsings with cold water will then remove nearly every particle of fat from the flask, which is fixed in an inverted position over the filter, and the whole

* The approximate number of drops will, of course, depend on the temperature of the fat at the time of weighing. 
allowed to stand overnight. Next morning the flask is found to be quite dry, and a small quantity of ether will easily remove the traces of fat adhering to it. The fatty acids on the filter will be found to be pretty well air-dried, and can be readily removed to a tared flask, having a tolerably wide mouth. The rinsings of ether from the saponifying flask are then poured over the paper filter, and fresh ether poured over it till, on drying, no trace of fat can be seen. If the fatty acids have been carefully and completely remored from the paper, previous to washing, a comparatively small quantity of ether will suffice for that purpose. The flask can then be attached to a condenser, and the ether nearly all recovered by distillation on a water bath, and a few drops of absolute alcohol haring been added to the flask containing the fat, the latter is further dried till constant in weight. As already stated, I. think it would be more advantageous to dry the fat by placing the flask on an open water bath. The use of a flask such as I have mentioned, having a short wide neck, and with the weight marked on with a diamond, is, I think, preferable to that of basins or flat dishes. The ether used can nearly all be quickly recovered, and although, from a pecuniary point of view, this may be no great object, yet it aroids the risk of ether vapours floating about, and is a much more conrenient method of getting rid of that agent. 'The fat does not rise up the sides of a flask, as it always does when flat dishes are employed, and the risk of a slight loss from this cause is avoided.

In order to test the process, a sample of fresh butter was procured from a farm near Greenock. This farm, it may be stated, is somewhat celebrated for the quality of the butter made there. On examination this sample gave the following results.

The sp. gr. of the dried and filtered fat was found to be 912.94 at $100^{\circ} \mathrm{F}$. Analysis gave :-

\begin{tabular}{|c|c|c|c|c|c|c|}
\hline \multirow{3}{*}{\multicolumn{2}{|c|}{$\begin{array}{l}\text { Soluble Fatty Acids } \\
\text { Insoluble },\end{array}$}} & & & & \multirow{2}{*}{\multicolumn{2}{|c|}{$\begin{array}{l}\text { Average. } \\
\ldots \quad 4 \cdot 74 \text { per cent. }\end{array}$}} \\
\hline & & & $\ldots$ & $\ldots$ & & \\
\hline & & $\ldots$ & $\ldots$ & $\ldots$ & $\ldots$ & $89 \cdot 37$ \\
\hline Total & $"$ & $\ldots$ & $\ldots$ & .• & $\ldots$ & 94.11 per cent. \\
\hline
\end{tabular}

A sample of beef fat was also obtained, the fat from which, when dried and filtered, had a sp. gr. of $902 \cdot 1$ at $100^{\circ} \mathrm{F}$. Four different mixtures of beef and butter fats were made up in the following proportions :-

$\begin{array}{lcccc} & \text { No. } 1 & 2 & 3 & 4 \\ \text { Butter Fat } & 52 \cdot 38 & 24 \cdot 69 & 96 \cdot 94 & 9900 \\ \text { Beef Fat } & 47 \cdot 62 & 75 \cdot 31 & 3 \cdot 06 & 1 \cdot 00\end{array}$

Analysis of these mixtures gave the following results :-

\begin{tabular}{|c|c|c|c|c|}
\hline & No. 1 & 2 & 3 & 4 \\
\hline luble Acids $; \mathrm{F}$ & $\begin{array}{l}2 \cdot 39 \\
2 \cdot 48\end{array}$ & $\begin{array}{r}\cdot 95 \\
1 \cdot 17\end{array}$ & $\begin{array}{l}4 \cdot 50 \\
4 \cdot 59\end{array}$ & $\begin{array}{l}4 \cdot 6 \\
4 \cdot 6\end{array}$ \\
\hline soluble Acids fo & 93.37 & 94.94 & 90.76 & $89 \cdot 6$ \\
\hline
\end{tabular}

A sample of butter sent to me for analysis, and which was afterwards admitted to be adulterated, gave the undernoted results :-

The sp. gr. of the dried fat was 906.1 at $100^{\circ} \mathrm{F}$.

\begin{tabular}{|c|c|c|}
\hline Soluble Acids (as butyric & $\begin{array}{l}2 \cdot 20 \\
2 \cdot 17\end{array}$ & $2 \cdot 18$ per cent \\
\hline \multirow[t]{2}{*}{ Insoluble , } & $\begin{array}{l}91 \cdot 89 \\
92 \cdot 18\end{array}$ & $92 \cdot 03$ \\
\hline & & 94.21 per cent. \\
\hline
\end{tabular}


Taking a standard of 5 per cent. soluble acids, and calculating to the origina butter, gives :-

$$
\begin{array}{llllll}
\text { Butter Fat } & \ldots & \ldots & \ldots & \ldots & 38 \cdot 32 \text { per cent. } \\
\text { Foreign Fat } & \ldots & \ldots & \ldots & \ldots & 48 \cdot 76 \%
\end{array}
$$

The above results corroborate what, I think, has already "been pretty clearly established, viz., that a careful determination of the soluble "fatty acids" affords a very correct basis for judging of the amount of foreign fat present in any sample of adulterated butter; the addition of even 1 per cent. of beef fat showing an appreciable difference in their amount, and that, with ordinary care, the present method is capable of yielding very concordant results.

My best thanks are due to my friend Mr. Gossman, who kindly undertook the greater part of the work connected with these experiments.

In the discussion on the above two papers on Butter Analysis,

Dr. Dupré said that with regard to Mr. Jones' paper, it was unfortunate that he had published two analyses of some of the butters mentioned. In his first paper he found all his insoluble fatty acids very high. It was suggested by some of the members that he had not washed the fatty acids sufficiently, and therefore had left some of the soluble fatty acids in the insoluble. Mr. Jones, with great moral courage, came forward at the next meeting and said he had not believed it had been the case, but he had made some experiments and found it correct, and the difference in the results (which Dr. Dupré specified,) of course threw the whole of the table out and made it useless. The only thing useful was the sp. gr., which was not likely to have been affected, and as far as they went they corresponded very much with some observations of his own. He had found that some butters did not vary much in two years and a half, while others changed very rapidly. The changes which butter undergoes depended greatly upon the character of the original butter, and it was evidently impossible to say at any time what a butter might be a year hence. With regard to Dr. Milne's paper he could not see the use of once putting the fatty acids in a flask and then taking them out, but having once put them in never take them out, and then there was no chance of losing any drop, or of any other difficulties. He held, and held very strongly, every butter analyses to be worthless that gave the fat as much above 98 per cent., that is the fatty acids plus glycerine residue. Taking the average of the ten analyses given in his (Dr. Duprés) paper on butter, they came to 97.99 per cent. of fat.-taking the equivalent of the insoluble fatty acids at 275 , and the soluble fatty acids as butyric acid at 88 . Taking the four butters Mr. Jones had re-analysed, the mean was 98.05 , and taking the four acids according to his first paper-admittedly incorrect-the fat came to 98.75. In Dr. Milne's paper the fat comes to $99 \cdot 28$. Dr. Dupré had no hesitation in saying that no analysis with a high percentage of insoluble fatty acids should be accepted, unless it was accompanied by the statement of the percentage of soluble fatty acids.

$\mathrm{Mr}$. Hehner pointed out that the fact that fat increased in weight when kept melted at $100^{\circ}$, was already known. He further remarked, that in most of Mr. Jones' analyses the difference between the insoluble acids on the first and second analysis was small. He found on comparison, that in the soluble acids three had risen and six had fallen-that was assuming the analyses to be correct. If the soluble fatty acids had fallen, then there was very great risk of confounding a genuine butter with an adulterated one. Looking at the insoluble fatty acids three had risen, and two of those very inconsiderably, quite 
within the limits of error. Whilst it was possible that an adulterated butter might pass as genuine, he thought it was important that they should not condemn a genuine butter, but much rather let a bad one pass. In some cases the butters had changed considerably, in nearly all cases the fatty acids had gone in a right direction, and there was really no chance of confounding a genuine butter with an adulterated one. He thought Mr. Jones' figures were calculated to lead analysts into confusion.

The President having thanked the authors of the papers stated that he agreed with most of the remarks made by Dr. Dupré and Mr. Hehner upon them. 